First version: September 1997

This version: October 1999

\title{
On the Relevance of Modeling Volatility for Pricing Purposes
}

\author{
Manuel Moreno* \\ Department of Economics and Business \\ Universitat Pompeu Fabra \\ Carrer Ramón Trias Fargas, 25-27 \\ 08005 Barcelona, Spain. \\ phone: (34-93) 5.42.27.71 \\ fax: (34-93) 5.42.17.46 \\ e-mail: manuel.moreno@econ.upf.es.
}

\begin{abstract}
:
This paper presents a two-factor (Vasicek-CIR) model of the term structure of interest rates and develops its pricing and empirical properties. We assume that default free discount bond prices are determined by the time to maturity and two factors, the long-term interest rate and the spread. Assuming a certain process for both factors, a general bond pricing equation is derived and a closed-form expression for bond prices is obtained. Empirical evidence of the model's performance in comparisson with a double Vasicek model is presented. The main conclusion is that the modeling of the volatility in the long-term rate process can help (in a large amount) to fit the observed data can improve - in a reasonable quantity - the prediction of the future movements in the medium- and long-term interest rates. However, for shorter maturities, it is shown that the pricing errors are, basically, negligible and it is not so clear which is the best model to be used.
\end{abstract}

Keywords: Term Structure of Interest Rates, Bond Pricing Equation, TwoFactor Models, Ornstein-Uhlenbeck Process, CIR Process.

JEL classification: C51, E43, G13. 


\section{Introduction}

The evolution over time of interest rates for default-free zero-coupon bonds is a topic that has been extensively analyzed in the financial literature. Initially, the analysis of this evolution was performed by means of one-factor models which assume that movements in interest rates are driven by changes in the short-term (instantaneous) riskless interest rate (see, among others, Vasicek (1977), Cox et al (1985) or Chan et al (1992)). However, it is now widely accepted that interest rates are affected by more than one state variable. In this direction, several papers as Richard (1978), Brennan and Schwartz (1979), Schaefer and Schwartz (1984), Cox et al (1985), Longstaff and Schwartz (1992), Duffie and Kan (1996), Chen (1996), Dai and Singleton (1997) and Boudoukh et al (1999) use multiple factors to explain the future movements that interest rates may show.

There is substantial empirical evidence ${ }^{1}$ that shows that movements in interest rates can be decomposed in three types of "basic" changes related to the level of interest rates, the slope, and the curvature of the yield curve. As the curvature is usually the less important explanatory variable when dealing with spot interest rates, we can think that movements in spot interest rates may be reasonably well explained by the two first factors.

In fact, this is the motivation for the model previously presented and developed in Moreno (1996) which uses the long-term interest rate and the spread of interest rates as state variables (that is, the difference between the long-term interest rate and the short-term interest rate is used as a rough measure of the slope of the yield curve). In that paper, both factors are assumed to follow a Vasicek process and, therefore, both variables (1) show mean reversion to a certain long term value and (2) their diffusions reflect a constant variance term. Under these assumptions, a general bond pricing equation was derived and a closed-form expression for zero-coupon bond and for interest rate derivatives prices was computed, This paper also presented the empirical performance of this model in relation to an alternative onefactor model.

It can be argued that one of the assumptions made in Moreno (1996), namely, the constant variance in the diffusion of the processes followed by

\footnotetext{
${ }^{1}$ See, for instance, Jones (1991), Litterman and Scheinkman (1991), Zhang (1993) and Knez, Litterman, and Scheinkman (1994).
} 
both factors is too restrictive from an empirical point of view. ${ }^{2}$ This restrictive feature leads to the present paper whose main objective is to analyze if modeling the volatility improves the empirical performance of the Moreno (1996) model. Thus, the same state variables will be used although we will assume that the long-term interest rate does not follow a Vasicek process but a root-square (CIR-type) process. This alternative model will be denoted hereafter as the Vasicek-CIR model and it can be considered, from a theoretical point of view, as an special case of the Schaefer and Schwartz (1984) model.

The schedule of this paper is as follows. Section 2 presents the main assumptions of the Vasicek-CIR model and provides the basic pricing equation that any derivative asset must satisfy. This equation, with the appropriate terminal condition, allows us to obtain the price of any asset that, at maturity, pays a certain payoff as indicated in such terminal condition. In this section we (a) compute the analytical expression that indicate the price of any discount bond under the assumptions given by this two-factor model and (b) recall the analogous formula that was obtained in Moreno (1996) (Vasicek-Vasicek model hereafter). Section 3 analyzes the empirical behavior of both models by comparing the usefulness of these alternative formulas to fit and forecast bond prices, that is, the in- and out-of-sample performance of such expressions. The data analyzed correspond to Spanish interest rates and bond prices for different maturities during the period 1991-1995. Finally, Section 4 summarizes and concludes.

\section{The Bond Pricing Equation}

In this section we present the two-factor (Vasicek-CIR) model that we will use to price default-free discount bonds by deriving (and solving) the pricing equation which must be verified by the prices of these bonds.

The main assumption of this model is that the price, at time $t$, of a default-free discount bond that pays $\$ 1$ at maturity $T$ depends only on the current values of two state variables and time to maturity, $\tau=T-t$. The

\footnotetext{
${ }^{2}$ Interest rate volatility is usually increasing in interest rate level although there is no consensus about the exact relationship between volatility and level. See Chan et al (1992), Aït-Sahalia (1996), Conley et al (1997) and Stanton (1997) for this issue.
} 
main motivation for the factors to be used is the empirical evidence (see footnote 1) that changes in interest rates are a combination of movements in (a) the level of interest rates, (b) the slope and (c) the curvature of the yield curve, which effect is usually negligible. Therefore, we can use the long-term rate and the spread as the variables that help us to explain the movements in the general level of interest rates and changes in the relationship between the short and the long end of the yield curve. With both variables, we can also try to explain the intermediate movements of the yield curve. ${ }^{3}$

Although most previous studies use the short-term interest rate as one of the state variable, we redefine these variables and, analogously to Schaefer and Schwartz (1984), the factors to be used are the long-term rate, denoted by $L$, and the spread, denoted by $s$, the difference between the long- and the short-term rate, denoted by $r$. This selection of state variables allows us to use the assumption of orthogonality between them. ${ }^{4}$

Once chosen these variables, we assume that their evolution over time is given by the following stochastic differential equations ${ }^{5}$ :

$$
\left\{\begin{aligned}
d s & =\beta_{1}(s, L) d t+\sigma_{1}(s, L) d w_{1} \\
d L & =\beta_{2}(s, L) d t+\sigma_{2}(s, L) d w_{2}
\end{aligned}\right.
$$

where $t$ denotes calendar time, and $d w_{1}$ and $d w_{2}$ are standard Brownian processes where $E\left[d w_{1}\right]=E\left[d w_{2}\right]=0, d w_{1}^{2}=d w_{2}^{2}=d t$, and (by the orthogonality assumption) it is verified that $E\left[d w_{1} d w_{2}\right]=0 . \beta_{1}($.$) and \beta_{2}($.$) are the$ expected instantaneous rates of change in the state variables and $\sigma_{1}^{2}($.$) and$ $\sigma_{2}^{2}($.$) are the instantaneous variances of changes in these factors.$

Let $P(s, L, t, T) \equiv P(s, L, \tau)$ be the price, at time $t$, of a default-free discount bond that pays $\$ 1$ at maturity $T=t+\tau$. We can express the instantaneous percentage change in the price of this bond as the sum of its expected rate of return and the unexpected variations in return due to the

\footnotetext{
${ }^{3}$ Two alternative couples of factors to be used may be: (a) the long-term interest rate and the short-term interest rate and (b) the short-term interest rate and the spread. However, the above two variables are chosen because of a better analytical tractability.

${ }^{4}$ This assumption simplifies the analytical tractability of the model. Empirical evidence that supports this assumption can been seen in Ayres and Barry (1980), Schaefer (1980), Nelson and Schaefer (1983) and, for the Spanish case, in Moreno (1996).

${ }^{5}$ After presenting this generic model and deriving the general pricing equation, we will particularize it to obtain the Vasicek-CIR model
} 
random changes in the factors

$$
\frac{d P(s, L, t, T)}{P(s, L, t, T)}=\mu(s, L, t, T) d t+s_{1}(s, L, t, T) d w_{1}+s_{2}(s, L, t, T) d w_{2}
$$

The steps to be given to obtain the bond pricing equation are very standard ${ }^{6}$ and can be summarized as follows:

1. Application of Itô's Lemma

2. Setting up of a (hedging) portfolio, composed of three bonds with different maturities, that is instantaneously riskless

3. Under no-arbitrage conditions, the expected rate of return of this portfolio must equate the instantaneous riskless rate of interest

These three steps jointly with a little algebra lead us to the following partial differential equation

$$
\begin{aligned}
& \frac{1}{2}\left[\sigma_{1}^{2}(.) P_{s s}+\sigma_{2}^{2}(.) P_{L L}\right]+\left[\beta_{1}(.)-\lambda_{1}(.) \sigma_{1}(.)\right] P_{s} \\
& +\left[\beta_{2}(.)-\lambda_{2}(.) \sigma_{2}(.)\right] P_{L}+P_{t}-r P=0
\end{aligned}
$$

where subscripts denote partial derivatives. The coefficients $\lambda_{1}($.$) and \lambda_{2}($. can be interpreted as the market prices of the spread and long-term rate risk, respectively.

Therefore, given the stochastic process (1) we have assumed for both variables, (3) is the fundamental equation for the pricing of default-free discount bonds of different maturities which depend solely on the spread, the longterm interest rate, and its time to maturity. In this equation we deal with the market prices of risk, $\lambda_{i}($.$) , because the only way to tie down the bond$ prices in our (partial equilibrium) model is by means of these (exogenous) parameters.

The solution of the equation (3), subject to the terminal condition given by the final payment of the bond, $P(s, L, 0)=1, \forall s, L$, is the price of the discount bond we are looking for.

\footnotetext{
${ }^{6}$ For more details, see Moreno (1996).
} 
The coefficients of the bond pricing equation (3) are the parameters of the stochastic process (1) which was assumed for the two factors and the market prices of the risk related to both state variables. As this equation is too general to be solved analytically, we will make the following assumptions about these coefficients:

Assumption 1 The market price of the spread risk is linear in this variable, that is

$$
\lambda_{1}(.)=a+b s
$$

Assumption 2 The market price of the long-term rate risk is proportional to the square root of this variable, that is

$$
\lambda_{2}(.)=d \sqrt{L}
$$

Assumption 3 Each of the state variables follow a diffusion process

$$
\left\{\begin{array}{l}
d s=k_{1}\left(\mu_{1}-s\right) d t+\sigma_{1} d w_{1} \\
d L=k_{2}\left(\mu_{2}-L\right) d t+\sigma_{2} \sqrt{L} d w_{2}
\end{array}\right.
$$

The motivation for the first two assumptions is that a constant market price of risk is too restrictive and quite unrealistic. The first assumption is the generalization of the one presented in Vasicek (1977) while the second one is similar to the one obtained in Cox et al (1985). Regarding the third assumption, the first process, known as Ornstein-Uhlenbeck process, has been used previously by Vasicek (1977) while the second one was proposed in Cox et al (1985). Both processes show mean reversion, an important stylized fact that interest rates usually show. In the process assumed for the spread, we find a constant variance in the diffusion term while the variance of the longterm rate is proportional to its level. For each state variable, $k_{i}>0$ is the coefficient of mean reversion which reflects the speed of adjustment of the variable towards its long-run mean value, $\mu_{i}$, and $d w_{i}$ are standard Brownian motions.

Under these three assumptions, we can rewrite the equation (3) as

$$
\frac{1}{2} \sigma_{1}^{2} P_{s s}+q_{1}\left(\hat{\mu}_{1}-s\right) P_{s}+\frac{1}{2} \sigma_{2}^{2} L P_{L L}+q_{2}\left(\hat{\mu}_{2}-L\right) P_{L}+P_{t}-(L+s) P=0
$$


subject to the terminal condition

$$
P(s, L, 0)=1, \forall s, L
$$

where

$$
\begin{array}{ll}
q_{1}=k_{1}+b \sigma_{1}, & \hat{\mu}_{1}=\left(k_{1} \mu_{1}-a \sigma_{1}\right) / q_{1} \\
q_{2}=k_{2}+d \sigma_{2}, & \hat{\mu}_{2}=k_{2} \mu_{2} / q_{2}
\end{array}
$$

Solving the partial differential equation (5) we obtain the following proposition:

Proposition 1 The value at time t of a discount bond that pays $\$ 1$ at time $T, P(s, L, t, T) \equiv P(s, L, \tau)$, is given by

$$
P(s, L, t, T)=A(\tau) e^{-B(\tau) s-C(\tau) L}
$$

where $\tau=T-t$ and

$$
\begin{aligned}
A(\tau) & =A_{1}(\tau) A_{2}(\tau) \\
A_{1}(\tau) & =\exp \left\{-\frac{\sigma_{1}^{2}}{4 q_{1}} B^{2}(\tau)+s^{*}(B(\tau)-\tau)\right\} \\
A_{2}(\tau) & =\left[\frac{2 \gamma \exp \left\{\left(q_{2}+\gamma\right) \frac{\tau}{2}\right\}}{\left(q_{2}+\gamma\right) \exp \{\gamma \tau\}+\left(\gamma-q_{2}\right)}\right]^{2 k_{2} \mu_{2} / \sigma_{2}^{2}} \\
B(\tau) & =\frac{1-e^{-q_{1} \tau}}{q_{1}} \\
C(\tau) & =\frac{2(\exp \{\gamma \tau\}-1)}{\left(q_{2}+\gamma\right) \exp \{\gamma \tau\}+\left(\gamma-q_{2}\right)}
\end{aligned}
$$

with

$$
\begin{array}{lll}
q_{1}=k_{1}+b \sigma_{1}, & \hat{\mu}_{1}=\left(k_{1} \mu_{1}-a \sigma_{1}\right) / q_{1}, & s^{*}=\hat{\mu}_{1}-\sigma_{1}^{2} /\left(2 q_{1}^{2}\right) \\
q_{2}=k_{2}+d \sigma_{2}, & \hat{\mu}_{2}=k_{2} \mu_{2} / q_{2}, & \gamma=\sqrt{q_{2}^{2}+2 \sigma_{2}^{2}}
\end{array}
$$

Proof: See Appendix. 
The terms in equation (8) verify

$$
\begin{array}{lll}
0<A_{i}(\tau)<1, \forall \tau>0, & A_{i}(0)=1, & A_{i}(\infty)=0, i=1,2 \\
0<B(\tau)<\tau, \forall \tau>0, & B(0)=0, & B(\infty)=1 / q_{1} \\
0<C(\tau)<\tau, \forall \tau>0, & C(0)=0, & C(\infty)=2 /\left(q_{2}+\gamma\right)
\end{array}
$$

Substituting $t=T$ into $(7)$, it is shown that the terminal condition for the price bond, $P(s, L, 0)=1, \forall s, L$, is satisfied. Moreover, it is also derived that

$$
P(0,0, \tau)=A(\tau)=A_{1}(\tau) A_{2}(\tau)<1, \forall \tau>0
$$

It can be checked that the following realistic features are verified

$$
\lim _{s \rightarrow \infty} P(s, L, \tau)=\lim _{L \rightarrow \infty} P(s, L, \tau)=\lim _{\tau \rightarrow \infty} P(s, L, \tau)=0
$$

that is, when any of the arguments included in the bond price formula tends to infinity, the price converges to zero. It is also easily shown that the bond price function is decreasing and convex in both factors and decreasing with the time to maturity.

Once we have obtained the expression (and properties) for the bond price formula under the Vasicek-CIR model, we will recall the assumptions made in Moreno (1996) and the corresponding pricing formula that was derived in that paper:

Assumption 1' (equal to Assumption 1) The market price of the spread risk is linear in this variable, that is

$$
\lambda_{1}(.)=a+b s
$$

Assumption 2' The market price of the long-term rate risk is linear in this variable, that is

$$
\lambda_{2}(.)=c+d L
$$

Assumption 3' Each of the state variables follow a diffusion process of Vasicek type

$$
\left\{\begin{array}{l}
d s=k_{1}\left(\mu_{1}-s\right) d t+\sigma_{1} d w_{1} \\
d L=k_{3}\left(\mu_{3}-L\right) d t+\sigma_{3} d w_{3}
\end{array}\right.
$$

Under these assumptions, we can rewrite the equation (3) as

$$
\frac{1}{2} \sigma_{1}^{2} P_{s s}+q_{1}\left(\hat{\mu}_{1}-s\right) P_{s}+\frac{1}{2} \sigma_{3}^{2} P_{L L}+q_{3}\left(\hat{\mu}_{3}-L\right) P_{L}+P_{t}-(L+s) P=0
$$


where

$$
\begin{array}{ll}
q_{1}=k_{1}+b \sigma_{1}, & \hat{\mu}_{1}=\left(k_{1} \mu_{1}-a \sigma_{1}\right) / q_{1} \\
q_{3}=k_{3}+d \sigma_{3}, & \hat{\mu}_{3}=\left(k_{3} \mu_{3}-c \sigma_{3}\right) / q_{3}
\end{array}
$$

The solution of the differential equation (12), subject to the terminal condition given by the payoff of the bond at maturity (see equation (6)), was established in the following proposition:

Proposition 2 (Proposition 1 in Moreno (1996)) The value at time $t$ of a discount bond that pays $\$ 1$ at time $T, P(s, L, t, T) \equiv P(s, L, \tau)$, is given by

$$
P(s, L, \tau)=D(\tau) e^{-E(\tau) s-F(\tau) L}
$$

where $\tau=T-t$ and

$$
\begin{aligned}
D(\tau) & =D_{1}(\tau) D_{3}(\tau) \\
D_{1}(\tau) & =\exp \left\{-\frac{\sigma_{1}^{2}}{4 q_{1}} B^{2}(\tau)+s^{*}(B(\tau)-\tau)\right\} \\
D_{3}(\tau) & =\exp \left\{-\frac{\sigma_{3}^{2}}{4 q_{3}} C^{2}(\tau)+L^{*}(C(\tau)-\tau)\right\} \\
E(\tau) & =\frac{1-e^{-q_{1} \tau}}{q_{1}} \\
F(\tau) & =\frac{1-e^{-q_{3} \tau}}{q_{3}}
\end{aligned}
$$

with

$$
\begin{aligned}
& q_{1}=k_{1}+b \sigma_{1}, \quad \hat{\mu}_{1}=\left(k_{1} \mu_{1}-a \sigma_{1}\right) / q_{1}, \quad s^{*}=\hat{\mu}_{1}-\sigma_{1}^{2} /\left(2 q_{1}^{2}\right) \\
& q_{3}=k_{3}+d \sigma_{3}, \quad \hat{\mu}_{3}=\left(k_{3} \mu_{3}-c \sigma_{3}\right) / q_{3}, \quad L^{*}=\hat{\mu}_{3}-\sigma_{3}^{2} /\left(2 q_{3}^{2}\right)
\end{aligned}
$$

Proof: It is similar to the proof of Proposition 1 and it is omitted for the sake of brevity. 


\section{Empirical Application}

In this section, we describe the empirical application in which we compare the fitting and forecasting behavior of the Vasicek-CIR and the Vasicek-Vasicek models. This comparison is performed analyzing the in- and out-of-sample properties of both models. The dataset consists of daily Spanish interest rates and zero-coupon bond prices and cover the period 1991-1995. ${ }^{7}$

For each day of this period, we have interest rates (in annualized form) and bond prices for ten different maturities: 1, 7, and 15 days, 1, 3, and 6 months, and 1, 3, 5, and 10 years. The interest rates corresponding to the shortest and longest maturity (1 day / 10 years) are used as proxies of the short- and long-term interest rate, respectively.

The main descriptive characteristics of the state variables used in both two-factor models are:

1. For both interest rate series, the unconditional average is larger than $10 \%$. Short-term interest rates are larger than this mean value until October 1993 while the long-term interest rates exceed this level in the whole period except from June 1993 through June 1994. On the other hand, the spread has a mean value very close to zero and ranges between $-4 \%$ and $8 \%$.

2. The short-term rate is more volatile and moves into a wider interval than long-term rates do.

3. Both state variables show an uniformly high degree of serial correlation.

4. Most of the changes in the short-term interest rates are smaller than 100 basis points while changes in long-term rates are much smoother. As a consequence, changes in the spread are quite similar to changes in short-term interest rates.

5. It is seen a small decrease - in mean - in interest rates through the sample period.

6. Evidence of mean reversion in spread and interest rates is derived.

\footnotetext{
${ }^{7}$ For more details on these data, see Nuñez (1995) for technical details on the procedure used by the Bank of Spain to estimate them and Moreno (1996) for a descriptive and graphical analysis.
} 
7. The theoretical assumption about the orthogonality between the state variables is empirically corroborated.

Next we present the empirical performance of both models. We recall that both models use the same factors and the main difference between them derives from the alternative processes assumed for the long-term rate, $L$.

Each state variable of the two competing models, $s$ and $L$, follows a diffusion process (see equations (4) and (11)). The diffusion parameters of these processes $\left(k_{i}, \mu_{i}, \sigma_{i}, i=1,2,3\right)$ are estimated by the Generalized Method of Moments presented in Hansen $(1982)^{8}$. The econometric specification in discrete time is

$$
\begin{aligned}
s_{t}-s_{t-1} & =a_{1}+b_{1} s_{t-1}+\varepsilon_{t}^{s}, & & \varepsilon_{t}^{s} \sim \operatorname{IID}\left(0, \sigma_{1}^{2}\right) \\
L_{t}-L_{t-1} & =a_{2}+b_{2} L_{t-1}+\varepsilon_{t}^{L}, & & \varepsilon_{t}^{L} \sim \operatorname{IID}\left(0, \sigma_{2}^{2} r_{t-1}\right) \\
L_{t}-L_{t-1} & =a_{3}+b_{3} L_{t-1}+\varepsilon_{t}^{L}, & & \varepsilon_{t}^{L} \sim \operatorname{IID}\left(0, \sigma_{3}^{2}\right)
\end{aligned}
$$

so that

$$
k_{i}=-b_{i}, \quad \mu_{i}=-\frac{a_{i}}{b_{i}}, \quad i=1,2,3
$$

Table I includes the estimation results obtained for the sample period 1991-1995 and shows that the parameters $b_{i}$ of the discrete time specification (and hence, the diffusion parameters $k_{i}$ ) are significantly different from zero. So, there is evidence of mean reversion in all the state variables.

In both models, the long-term interest rate tends to a mean value close to $10 \%$ while the spread tends to a mean value close to zero. Comparing the two processes assumed for the long-term rate, it may be interesting to recognize that, under the CIR model, the long rate reverts faster to its long-term value than when considering the Vasicek assumption.

After estimating the parameters of the diffusion processes followed by the factors in both models, these values are used to obtain the remaining parameters of equations (7) and (13). Thus, similarly to Moreno (1996), we use the specifications

$$
\begin{aligned}
& P=P\left(q_{1}, q_{2}, s^{*} \mid k_{1}, k_{2}, \mu_{1}, \mu_{2}, \sigma_{1}, \sigma_{2} ; s, L, \tau\right)+\varepsilon \\
& P=P\left(q_{1}, q_{3}, s^{*}, L^{*} \mid k_{1}, k_{3}, \mu_{1}, \mu_{3}, \sigma_{1}, \sigma_{3} ; s, L, \tau\right)+\varepsilon
\end{aligned}
$$

\footnotetext{
${ }^{8}$ For details on this technique and its applications in the estimation of continuous-time models, see Moreno and Peña (1996).
} 
where $P$ is the observed price of the discount bonds available at time $t, P($. is the closed-form pricing equation for each model (see equations (7) and (13)) and $\varepsilon$ is an error term.

The parameters of the equations $(16)\left(q_{i}, i=1,2,3, s^{*}, L^{*}\right)$ are estimated on a daily basis for the period 1991-1995 by means of a panel of data where we have daily yield curves containing a cross-section of discount bond prices. Therefore, we have a matrix with 1230 rows and 10 columns where each row includes the (ten) zero-coupon bond prices available at each day and each column contains the bond prices for a certain maturity.

We estimate the non-linear equations (16) for each day of the period 1991-1995. The estimation of the first equation provides the parameters of the Vasicek-CIR model (that is, $q_{1}, q_{2}, s^{*}$ ) while the estimation procedure, when applied to the second equation, provides the parameters of the VasicekVasicek model, that is, $q_{1}, q_{3}, s^{*}, L^{*}$.

Estimation results for the daily parameters of the Vasicek-CIR model are included in Table II. ${ }^{9}$ This table shows the average of the estimated parameters obtained for (a) the full sample period and (b) the sample period divided year by year and reflects that all the parameters are positive and highly significant.

The evolution over time of these parameters can be seen graphically in Figure 1. This figure shows that the highest values are attained in 1991 while the lowest (and more stable) parameters correspond to the period 1994-1995.

In the next step, we can compute the values, day by day, of the market prices of risk related to each state variable using the estimated parameters obtained from equation (16) jointly with the expressions (9) and (15) and the Assumptions 1 and 2. A graphical representation of these values, for the Vasicek-CIR model, can be seen in Figure 2. The average values of these prices - under both models - for the whole period and for every year, are included in Table III.

Analyzing the two factors of the Vasicek-CIR model, both market prices of risk are highly significant and have a similar behavior across the period 1991-1995: each price has always the same sign during all the period 19911995 (for the long-term rate, the market price of risk is always positive and

\footnotetext{
${ }^{9}$ We do not show the results for the Vasicek-Vasicek model that can be seen in Table VIII in Moreno (1996). That paper provided all the results for the Vasicek-Vasicek model that are included in the following tables in this paper.
} 
the risk related to the spread has always a negative price) and both series of market prices are specially low in 1992 and the second half of the period 19911995. In absolute terms, the price of the spread risk is, at every moment, much higher than the price of risk of the long-term rate.

On the other hand, for the Vasicek-Vasicek model, we can observe a very different behavior between both factors and with respect to the alternative model. Thus, the two basic features for the prices of risk in this model are:

1. For the full period, the market prices of risk for both state variables are positive and significantly different from zero.

2. Analyzing this period year by year, the parameters are also significantly different from zero but they show a changing sign. Thus, the mean market price of risk of the spread is negative in the last two years of the sample period while the average of the market price of risk related to the long-term rate is negative in 1991-1992 and 1994.

Finally, we will use the values of the diffusion parameters jointly with the parameters estimated by means of the equation (16) to analyze the fitting and forecasting power of both two-factor models. The within- and out-of-sample periods are 1991-1994 and 1995, respectively.

First, the in-sample estimated data, for each day of the period 1991-1994 and for both models, are provided by the inclusion of the (daily) estimated parameters and the estimated parameters of the diffusion processes in the non-linear equation (16).

Next, we will compare the out-of-sample properties of both models by using the $k$-step-ahead forecasts that are generated for the bond prices. These $t+k$-time forecast values are built using the coefficient estimated from time t. This procedure is repeated for each day of 1995 .

Once obtained the in- and out-of-sample forecasts, the (within and outof-sample) pricing errors of both models are computed to compare one each other. Thus, we define, for time $t$, the error, $e_{t}$, and the percentage error, $P E_{t}$, as

$$
e_{t}=P_{t}-\hat{P}_{t}, \quad P E_{t}=\frac{P_{t}-\hat{P}_{t}}{P_{t}} \times 100
$$

where $P_{t}$ and $\hat{P}_{t}$ are, respectively, the observed and the estimated (fitted or forecasted) price, for time $t$, of the zero-coupon bond with a certain maturity. 
For both models, the within-sample (absolute and percentage) pricing errors are shown in Figures 3 and 4 . For all the maturities, it can be seen that the Vasicek-Vasicek model provides a very large pricing error in May, 1993. This error coincides with a sharp change in the short-term rate and in the spread. For both models, neither figure suggests a systematic pattern in these pricing errors.

Denoting by $N$ the number of days of the period to be analyzed, we use the pricing errors to compute several accuracy measures that help us to compare the empirical performance of both models:

1. Mean Error (ME). This measure weights equally the daily errors. Therefore, positive values can be offset with negative values and, thus, this measure may be small even with large errors. Its expression is

$$
M E=\frac{1}{N} \sum_{t=1}^{N} e_{t}=\frac{1}{N} \sum_{t=1}^{N}\left(P_{t}-\hat{P}_{t}\right)
$$

2. Mean Absolute Error (MAE). As the mean error, this measure gives an equal weight to the daily errors but positive and negative errors do not cancel out. It is defined as

$$
M A E=\frac{1}{N} \sum_{t=1}^{N}\left|e_{t}\right|=\frac{1}{N} \sum_{t=1}^{N}\left|P_{t}-\hat{P}_{t}\right|
$$

3. Root Mean Squared Error (RMSE). It is usually the most common measure of accuracy and its definition is

$$
R M S E=\sqrt{\frac{1}{N} \sum_{t=1}^{N}\left(e_{t}\right)^{2}}=\sqrt{\frac{1}{N} \sum_{t=1}^{N}\left(P_{t}-\hat{P}_{t}\right)^{2}}
$$

4. Mean Percentage Absolute Error (MAPE) Similarly to the mean absolute error, the absolute value of the error is used but each error is weighted by the current value of the bond price. Its expression is

$$
M A P E=\frac{1}{N} \sum_{t=1}^{N}\left|P E_{t}\right|
$$


5. Root Mean Squared Percentage Error (RMSPE). This measure is similar to the root mean squared error but, similarly to the MAPE, the daily errors are weighted by the actual bond prices. It is given by

$$
R M S P E=\sqrt{\frac{1}{N} \sum_{t=1}^{N}\left(P E_{t}\right)^{2}}
$$

These five descriptive measures, for both models, are computed for 19911994 (within sample period), for 1995 (out-of-sample period) and for different subperiods. The within and out-of-sample results are reported in Tables IVVI and Tables VII-X, respectively.

The performance of both models, for the within-sample period, is included in Table IV. For this period, the Vasicek-Vasicek model underprices the shortand medium-term bonds and overprices the bonds whose maturity is beyond one year. On the other hand, the Vasicek-CIR model overprices the bonds with maturities up to three months as well as the 5 -year bonds.

All the statistics included in Table IV reflect that both models provide a very big accuracy to the observed bond prices for all the maturities. It can be seen that the pricing errors are increasing with the maturities (the longer the maturity, the larger the error price) but the MAPE for the Vasicek-Vasicek and the Vasicek-CIR models is smaller than $0.26 \%$ and $0.02 \%$, respectively. Moreover, in the Vasicek-CIR (Vasicek-Vasicek) model, this statistic is always smaller than $0.01 \%$ (0.08\%) except for the 5 -year bond price.

Comparing both models, the Vasicek-CIR model outperforms the VasicekVasicek model for all maturities and for all the statistics. In short-term bonds, with maturities smaller than one month, both models provide negligible errors and the improvement obtained with the Vasicek-CIR model over the other one is not very large.

On the other hand, focusing on the maturities beyond one month, the Vasicek-CIR model provides a huge improvement: the errors from the VasicekVasicek model are decreased in more than $88 \%$ for all these maturities. The biggest improvement in accuracy is achieved in the medium-term maturities (six month and one year) and in 5-year bonds, maturity in which the error measures from the Vasicek-CIR model are about $6 \%$ of the error measures provided by the Vasicek-Vasicek model. This conclusion is obtained for all the statistics. 
Table $\mathrm{V}$ includes the results obtained for the year 1992. Similarly to the period 1991-1994, the Vasicek-Vasicek model underprices the shortest maturities (up to six months). In contrast to what happened in the whole within-sample period, this underpricing can also be seen in the Vasicek-CIR model which, on the other hand, overprices the bonds that mature beyond one year.

In this year, both models fit the observed data specially well. Thus, the error measures for both models are decreased in more than half for most of the maturities with respect to the whole period. Comparing both models, the error measures of the Vasicek-CIR model, as in the whitin-sample period, are about $10 \%$ of the statistics provided by the Vasicek-Vasicek model for all the maturities longer than 15 days.

Therefore, the main conclusion for this year is the same than the obtained for the period 1991-1994: for the shortest maturities, both models fit specially well to the data but, for most of the remaining maturities, the Vasicek-CIR model provides a remarkable large improvement in accuracy.

Several subperiods have been analyzed and, basically, the same conclusions are reached. For illustrative purposes, Table VI includes the whitinsample results obtained for 1-year bonds for each semester of the period 1991-1994.

Looking at every statistic, it is shown that the Vasicek-CIR model fits better than its competing model in all the semesters and it works specially well in 1991 and 1994 while the Vasicek-Vasicek model obtains its best performance in the first semester of 1992 and in the second one of 1994.

Based on a mean absolute error (MAE or MAPE) criterion, the superiority of the Vasicek-CIR model implies an improvement of about $90 \%$ in all the semesters but the last one in which the errors from the Vasicek-Vasicek model are decreased in 'just' $77 \%$.

For all the semesters, the mean absolute percentage error of the VasicekCIR model is around $0.003 \%$ that is about fifteen times smaller than the obtained for the Vasicek-Vasicek model. This superiority is specially remarkable in the second semester of 1991 and in the first one of 1994 when the absolute value of the errors are decreased in more than $96 \%$.

The forecasting power of both models is analyzed by computation of oneand five-step ahead forecasts ${ }^{10}$ of bond prices for every maturity and for every

\footnotetext{
${ }^{10}$ Ten-step-ahead forecasts were also computed. Results are available upon request.
} 
day of the year 1995. Measures of the forecasting pricing errors are included in Tables VII-X.

Tables VII-VIII include the measures for one-step-ahead forecasts. Thus, Table VII provides the one-step-ahead measures for the whole out-of-sample period. It can be seen that (1) both models forecast quite well, (2) the forecasting power decreases with the time to maturity, and (3) for both models, the MAPE (RMSPE) is always smaller than $0.36 \%(0.48 \%)$. It can also be seen that both models perform similarly for the shortest maturities and, in fact, the Vasicek-Vasicek model performs slightly better than the VasicekCIR model.

For maturities beyond one month, the Vasicek-CIR model forecasts better than the Vasicek-Vasicek model showing that the modeling of the volatility in the long-term rate process helps to predict the movements in the mediumand long-term interest rates.

This superior forecasting performance is not monotonic in the time to maturity. Thus, the largest improvement is obtained in the 1-year bond prices when the error measures from the Vasicek-Vasicek model are decreased in more than $21 \%(15 \%)$ when working on a (root) mean absolute criterion. In the remaining bonds, the improvement in the relative forecasting power is much smaller $(2-7 \%)$ and never exceeds $12 \%$, value that is obtained when forecasting the 5 -year bonds.

Table VIII provides the error measures obtained, for every month of 1995 , from 1-year bonds in which the Vasicek-CIR model achieves its best relative performance. Both models perform better in the second semester (with a MAPE (RMSPE) smaller than $0.06 \%(0.08 \%)$ ) than in the first one, when the MAPE (RMSPE) reaches $0.1 \%(0.12 \%)$. It can also be seen a similar behavior in both models from January to April (with a small superiority of the Vasicek-Vasicek model in this period) and in the two last months of 1995. In the period May-October, the Vasicek-CIR model achieves an improvement of the out-of-sample performance that ranges between $20 \%$ (in May) and $65 \%$ (in July).

Finally, Tables IX-X show the results obtained when the forecasting horizon is five days. Thus, Table IX includes the measures obtained with the out-of-sample errors for all the maturities in 1995. Although these measures are bigger than in the shorter forecasts, they are reasonably small as reflected in the MAPE or the RMSPE that are, for both models, smaller than $0.8 \%$ and $1 \%$, respectively. 
The results are analogous to the obtained with the previous predictions: (1) the forecasting power decreases with time to maturity, (2) the VasicekVasicek model outperforms its rival model in the maturities smaller than six months, and (3) the Vasicek-CIR forecasts better than the Vasicek-Vasicek model in the maturities beyond six months. However, this improvement is usually quite small (between $1 \%$ and $3 \%$ ) and only increases until $5 \%$ when forecasting 1-year bond prices.

The last table provides the quarterly results obtained with five-step-ahead forecasts for 1- and 5-year bonds. In both maturities, it can be seen a better forecasting behavior in the second half of 1995 than in the first one. For 1-year bonds, both models show a MAPE smaller than $0.2 \%$ in all the quarters and the Vasicek-CIR model outperforms the Vasicek-Vasicek model whose error measures are decreased between $5 \%$ and $15 \%$ in the period April-September.

Focusing on the forecasting errors for 5 -year bond prices, the MAPE statistic ranges between $0.6 \%$ and $1 \%$. In this case, the Vasicek-CIR model improves the forecasts from its competing model in $10 \%$ from July to September and, in the remaining quarters, its improvement is much smaller $(1-2 \%)$.

\section{Conclusions}

This paper has presented a two-factor (Vasicek-CIR) model in continuous time for the analysis of the terms structure of interest rates and its empirical behavior has been analyzed with respect to a second alternative model. The main common characteristic of these two models is that both employ the same factors (state variables) to explain the unexpected changes that interest rates may show in the future. These factors are the long-term interest rate and the spread, the difference between the long- and the short-term interest rate.

The Vasicek-CIR model assumes that the spread follows a Vasicek process while the long-rate is modeled as a CIR-type process. On the other hand, the Vasicek-Vasicek model has assumed that both variables follow a Vasicek process. This second model was previously presented Moreno (1996) which also developed its pricing properties, the implications on the term structure of interest rates and analyzed its empirical properties with a sample of daily interest rates and bond prices that cover the period 1991-1995.

The main objective of this paper is analyze and compare the empirical 
performance of both models in the period 1991-1995. Therefore, we can determine if modeling the volatility of the long-term interest rate may help us to explain the future movements of interest rates. As a starting point, we have derived a bond pricing equation whose solution indicates the price of a zero-coupon bond under certain assumptions, namely, this price depends solely on the current values of two state variables (mentioned above) and the time to maturity of the bond.

After this solution is obtained, it is used to analyze the fitting and forecasting properties of this model. These properties are, in a posterior stage, compared with the ones derived from the Vasicek-Vasicek model. The parameters of our competing models have been estimated in two steps. In the first one, the parameters of the diffusion processes have been estimated by the Generalized Method of Moments by Hansen (1982). Once these values are obtained, the remaining parameters are estimated by using a cross-section technique. As a result of this combination of estimation methods, we have been able to obtain the daily market prices of risk corresponding to both state variables for both models.

Thus, it has been shown that, for the Vasicek-CIR model, these prices are highly significant and have a constant sign during all the period 1991-1995. On the other hand, under the Vasicek-Vasicek model, it can be seen that the market prices of risk are significantly different from zero and positive for 1991-1995 although they show a changing sign when analyzing yearly this period.

Finally, we have analyzed the fitting and forecasting power of both models. The within- and out-of-sample periods are 1991-1994 and 1995, respectively. After computing the within- and out-of-sample forecasts, the pricing errors of both models (and several accuracy measures) for different subperiods have been obtained to compare one each other.

All these statistics show the following facts: (1) both models provide a very big accuracy to the observed bond prices for all the maturities, (2) the pricing errors are increasing with the maturities, (3) the MAPE for the Vasicek-Vasicek and the Vasicek-CIR models is smaller than $0.26 \%$ and $0.02 \%$, and (4) in the Vasicek-CIR (Vasicek-Vasicek) model, this statistic is always smaller than $0.01 \%(0.08 \%)$ except for the 5 -year bond price.

Comparing both models, it can be seen that (1) the Vasicek-CIR model outperforms the Vasicek-Vasicek model for all maturities and for all the statistics, (2) in maturities smaller than one month, both models provide 
negligible errors and the relative improvement obtained with the VasicekCIR model is not very large, (3) dealing with maturities greater than one month, the errors from the Vasicek-Vasicek model are decreased in more than $88 \%$ for all these maturities, (4) the biggest improvement (about $90-94 \%$ ) in accuracy is achieved in the medium-term maturities and in 5-year bonds. Several subperiods have been analyzed and the same conclusions are reached.

The forecasting power of both models has been analyzed by one- and fivestep ahead forecasts for every maturity and for every day of the year 1995 . Looking at one-step-ahead forecasts, it has been shown that - similarly to the within-sample period - both models perform quite well, the forecasting power decreases with the time to maturity and, for the shortest maturities, both models perform similarly. However, for maturities beyond one month, the Vasicek-CIR model forecasts better than the Vasicek-Vasicek model although this superior forecasting behavior is not monotonic in the time to maturity. Thus, the best relative performance is obtained in the 1-year bond prices when the error measures from the Vasicek-Vasicek model are decreased in more than $21 \%$. In the remaining bonds, the improvement is much smaller ranging between $2 \%$ and $12 \%$.

Finally, dealing with five-step-ahead forecasts, all the statistics reflect a worse performance than in the previous (shorter) forecasts although similar results are shown: (1) the forecasting power decreases with time to maturity, (2) the Vasicek-Vasicek model outperforms its rival model in the shortest maturities and (3) the Vasicek-CIR forecasts better than the Vasicek-Vasicek model in the maturities beyond six months. In this case, this improvement is usually quite small, between $1 \%$ and $5 \%$.

Therefore, the main conclusion is that the modeling of the volatility in the long-term rate process can help (in a large amount) to fit the observed data can improve - in a reasonable quantity - the prediction of the future movements in the medium- and long-term interest rates. However, for shorter maturities, it has been shown that the pricing errors are, basically, negligible and it is not so clear which is the best model to be used. 


\section{Appendix: Proofs}

\section{Proof of Proposition 1}

The method of the separation of variables allows us to write the solution of the equation (5) subject to (6) as

$$
P(s, L, t, T)=X(s, t, T) Z(L, t, T)
$$

where $X(s, t, T)$ solves the equation

$$
\frac{1}{2} \sigma_{1}^{2} X_{s s}+q_{1}\left(\hat{\mu}_{1}-s\right) X_{s}+X_{t}-s X=0
$$

subject to the terminal condition

$$
X(s, T, T)=1, \forall s
$$

and $Z(L, t, T)$ is the solution of the equation

$$
\frac{1}{2} \sigma_{2}^{2} L Z_{L L}+q_{2}\left(\hat{\mu}_{2}-L\right) Z_{L}+Z_{t}-L Z=0
$$

with terminal condition

$$
Z(L, T, T)=1, \forall L
$$

To solve equation (18), we posit a solution of the type

$$
X(s, t, T)=X(s, \tau)=A_{1}(\tau) e^{-B(\tau) s}
$$

Hence, the equation (18) becomes

$$
\frac{1}{2} \sigma_{1}^{2} B^{2}(\tau)-q_{1}\left(\hat{\mu}_{1}-s\right) B(\tau)-\left[\frac{A_{1}^{\prime}(\tau)}{A_{1}(\tau)}-B^{\prime}(\tau) s\right]-s=0
$$

where, from (19), the terminal conditions are given by

$$
A_{1}(0)=1, \quad B(0)=0
$$


Equation (23) is linear in the variable $s$ and, therefore, it becomes null when the corresponding coefficients are equal to zero. Hence, this equation is equivalent to the following system of first-order differential equations

$$
\begin{aligned}
& q_{1} B(\tau)+B^{\prime}(\tau)-1=0 \\
& \frac{1}{2} \sigma_{1}^{2} B^{2}(\tau)-q_{1} \hat{\mu}_{1} B(\tau)-\frac{A_{1}^{\prime}(\tau)}{A_{1}(\tau)}=0
\end{aligned}
$$

subject to the terminal conditions (24).

We first solve (25) with terminal condition $B(0)=0$. Including this solution in (26), integrating this equation, and using $A_{1}(0)=1$, we obtain

$$
\begin{aligned}
B(\tau) & =\frac{1-e^{-q_{1} \tau}}{q_{1}} \\
A_{1}(\tau) & =\exp \left\{-\frac{\sigma_{1}^{2}}{4 q_{1}} B^{2}(\tau)+s^{*}(B(\tau)-\tau)\right\}
\end{aligned}
$$

where

$$
s^{*}=\hat{\mu}_{1}-\sigma_{1}^{2} /\left(2 q_{1}^{2}\right)
$$

Replacing (27) into (22), we obtain the final expression for $X(s, t, T)$. In a similar way, to solve equation (20), we posit a solution of the type

$$
Z(L, t, T)=Z(L, \tau)=A_{2}(\tau) e^{-C(\tau) L}
$$

Hence, the equation (20) becomes

$$
\frac{1}{2} \sigma_{2}^{2} L C^{2}(\tau)-q_{2}\left(\hat{\mu}_{2}-L\right) C(\tau)-\left[\frac{A_{2}^{\prime}(\tau)}{A_{2}(\tau)}-C^{\prime}(\tau) L\right]-L=0
$$

where, from (21), the terminal conditions are given by

$$
A_{2}(0)=1, \quad C(0)=0
$$

As equation (29) is linear in the variable $L$, this equation is equivalent to the following system of first-order differential equations

$$
\begin{aligned}
& \frac{1}{2} \sigma_{2}^{2} C^{2}(\tau)+q_{2} C(\tau)+C^{\prime}(\tau)-1=0 \\
& q_{2} \hat{\mu}_{2} C(\tau)+\frac{A_{2}^{\prime}(\tau)}{A_{2}(\tau)}=0
\end{aligned}
$$


subject to the terminal conditions $(30)$.

We first solve (31). It is straightforward to show that this equation can be rewritten as

$$
-\frac{2}{\sigma_{2}^{2}} \frac{d C(\tau)}{\left(C(\tau)-c_{1}\right)\left(C(\tau)-c_{2}\right)}=d \tau
$$

where

$$
c_{1}=\frac{-q_{2}+\gamma}{\sigma_{2}^{2}}>0, \quad c_{2}=\frac{-q_{2}-\gamma}{\sigma_{2}^{2}}<0, \quad \gamma=\sqrt{q_{2}^{2}+2 \sigma_{2}^{2}}
$$

Integrating this equation and using the terminal condition $C(0)=0$, it is obtained that

$$
\frac{1}{\gamma} \ln \left(\frac{C(\tau)-c_{2}}{C(\tau)-c_{1}}\right)=\tau+\frac{1}{\gamma} \ln \left(\frac{c_{2}}{c_{1}}\right)
$$

and a little algebra leads to

$$
C(\tau)=\frac{2(\exp \{\gamma \tau\}-1)}{\left(q_{2}+\gamma\right) \exp \{\gamma \tau\}+\left(\gamma-q_{2}\right)}
$$

Once we know $C(\tau)$, we can solve the equation (32) or, equivalently

$$
k_{2} \mu_{2} C(\tau)+\frac{A_{2}^{\prime}(\tau)}{A_{2}(\tau)}=0
$$

Integrating, we have

$$
\ln \left[A_{2}(\tau)\right]=-k_{2} \mu_{2} \int C(\tau) d \tau+k_{A}
$$

Let $y=\exp \{\gamma \tau\}$. Then, more algebra gives

$$
\int C(\tau) d \tau=\frac{2}{\sigma_{2}^{2}}\left[\ln \left(\left(q_{2}+\gamma\right) \exp \{\gamma \tau\}+\left(\gamma-q_{2}\right)\right)-\left(q_{2}+\gamma\right) \frac{\tau}{2}\right]
$$

Replacing this expression in (34) and applying the condition $A_{2}(0)=1$, the final expression for $A_{2}(\tau)$ is given by

$$
A_{2}(\tau)=\left[\frac{2 \gamma \exp \left\{\left(q_{2}+\gamma\right) \frac{\tau}{2}\right\}}{\left(q_{2}+\gamma\right) \exp \{\gamma \tau\}+\left(\gamma-q_{2}\right)}\right]^{2 k_{2} \mu_{2} / \sigma_{2}^{2}}
$$

Including (33) and (35) into (28), we obtain the final expression for $Z(L, t, T)$. This expression, jointly with (22), gives the closed-form formula for the default-free discount bond prices for all maturities. 


\section{References}

[1] Ait-Sahalia, Y. (1996). Testing Continuous-Time Models of the Spot Interest Rate. Review of Financial Studies, 9, 2, 385-426.

[2] Ayres, H.R. and J.Y. Barry (1980). A Theory of the U.S. Treasury Market Equilibrium. Management Science, 26, 6, 539-569.

[3] Boudoukh, J., M. Richardson, R. Stanton and R.F. Whitelaw (1999). A Multifactor, Nonlinear, Continuous-Time Model of Interest Rate Volatility, mimeo, New York University.

[4] Brennan, M.J. and E.S. Schwartz (1979). A Continuous Time Approach to the Pricing of Bonds. Journal of Banking and Finance, $133-155$.

[5] Chan, K. C., G.A. Karolyi, F.A. Longstaff and A.B. Sanders (1992). An Empirical Comparison of Alternative Models of the Short-Term Interest Rate. Journal of Finance, 47, 3, 1209-1227.

[6] Chen, L. (1996). Interest Rate Dynamics, Derivatives Pricing, and Risk Management. Springer-Verlag, Berlin.

[7] Conley, T.G., L.P. Hansen, E.G. Luttmer, and J. Scheinkman (1997). Short-Term Interest Rates as Subordinated Diffusions. Review of Financial Studies, 10, 3, 525-577.

[8] Cox, J.C., J.E. Ingersoll and S.A. Ross (1985). A Theory of the Term Structure of Interest Rates. Econometrica, 53, 385-408.

[9] Dai, Q. and K. Singleton (1998). Specification Analysis of Affine Term Structure Models, mimeo, NYU and Stanford University.

[10] Duffie, D. and R. Kan (1996). A Yield-Factor Model of Interest Rates. Mathematical Finance, 6, 4, 379-406.

[11] Longstaff, F.A. and E.S. Schwartz (1992). Interest Rate Volatility and The Term Structure: A Two-Factor General Equilibrium Model. Journal of Finance, 47, 4, 1259-1282. 
[12] Nelson, J. and S.M. Schaefer (1983). The Dynamics of the Term Structure and Alternative Portfolio Immunization Strategies. In Innovations in Bond Portfolio Management: Duration Analysis and Immunization, eds. G.O. Bierwag, G.G. Kaufman, and A. Toevs, Greenwich, CT: JAI Press.

[13] Nuñez, S. (1995). Estimación de la Estructura Temporal de los Tipos de Interés en España: Elección entre Métodos Alternativos. Documento de Trabajo 9522. Banco de España.

[14] Richard, S.F. (1978). An Arbitrage Model of the Term Structure of Interest Rates. Journal of Financial Economics, 6, 33-57.

[15] Schaefer, S. and E.S. Schwartz (1984). A Two-Factor Model of the Term Structure: An Approximate Analytical Solution. Journal of Financial and Quantitative Analysis, 19, 4, 413-424.

[16] Vasicek, O. (1977). An Equilibrium Characterization of the Term Structure. Journal of Financial Economics, 5, 177-188. 


\section{Table I. Estimates of the Diffusion Parameters}

This table provides the parameter estimates (with t-values in parentheses) of the processes followed by each state variable. The sample period is from January 1991 to December 1995. The parameters are estimated by means of the Generalized Method of Moments applied to the following equations

$$
\begin{aligned}
s_{t}-s_{t-1} & =a_{1}+b_{1} s_{t-1}+\varepsilon_{t}^{s}, & & \varepsilon_{t}^{s} \sim \operatorname{IID}\left(0, \sigma_{1}^{2}\right) \\
L_{t}-L_{t-1} & =a_{2}+b_{2} L_{t-1}+\varepsilon_{t}^{L}, & & \varepsilon_{t}^{L} \sim \operatorname{IID}\left(0, \sigma_{2}^{2} r_{t-1}\right) \\
L_{t}-L_{t-1} & =a_{3}+b_{3} L_{t-1}+\varepsilon_{t}^{L}, & & \varepsilon_{t}^{L} \sim \operatorname{IID}\left(0, \sigma_{3}^{2}\right)
\end{aligned}
$$

\begin{tabular}{lccccc}
\hline Variable & $a$ & $b$ & $k$ & $\mu$ & $\sigma$ \\
\hline Spread & $-2.08 \times 10^{-6}$ & -0.01544 & 0.01544 & $-0.1347 \times 10^{-3}$ & 0.003467 \\
& $(-0.0210)$ & $(-3.0756)$ & $(3.0756)$ & $(-0.021)$ & \\
\hline Long-Term Rate & $1.079 \times 10^{-3}$ & -0.01042 & 0.01042 & 0.103532 & 0.070703 \\
(CIR process) & $(3.5991)$ & $(-3.6285)$ & $(3.6285)$ & $(33.2787)$ & \\
\hline Long-Term Rate & $0.732 \times 10^{-3}$ & -0.00728 & 0.00728 & 0.100574 & 0.001159 \\
(Vasicek process) & $(2.2968)$ & $(-2.3988)$ & $(2.3988)$ & $(20.881)$ & \\
\hline
\end{tabular}




\section{Table II. Averages of Pure Cross-Sectional Regressions}

This table contains the estimation results, for each day of the period 1991-1995, of the parameters $\left(q_{i}, i=1,2, s^{*}\right)$ in the closed-form pricing equation for the Vasicek-CIR model

$$
P(s, L, t, T)=P(s, L, \tau)=A(\tau) e^{-B(\tau) s-C(\tau) L}
$$

where

$$
\begin{aligned}
A(\tau) & =A_{1}(\tau) A_{2}(\tau) \\
A_{1}(\tau) & =\exp \left\{-\frac{\sigma_{1}^{2}}{4 q_{1}} B^{2}(\tau)+s^{*}(B(\tau)-\tau)\right\}, A_{2}(\tau)=\left[\frac{2 \gamma \exp \left\{\left(q_{2}+\gamma\right) \frac{\tau}{2}\right\}}{\left(q_{2}+\gamma\right) \exp \{\gamma \tau\}+\left(\gamma-q_{2}\right)}\right]^{2 k_{2} \mu_{2} / \sigma_{2}^{2}} \\
B(\tau) & =\frac{1-e^{-q_{1} \tau}}{q_{1}}, C(\tau)=\frac{2(\exp \{\gamma \tau\}-1)}{\left(q_{2}+\gamma\right) \exp \{\gamma \tau\}+\left(\gamma-q_{2}\right)}
\end{aligned}
$$

with

$$
\begin{array}{lll}
q_{1}=k_{1}+b \sigma_{1}, & \hat{\mu}_{1}=\left(k_{1} \mu_{1}-a \sigma_{1}\right) / q_{1}, & s^{*}=\hat{\mu}_{1}-\sigma_{1}^{2} /\left(2 q_{1}^{2}\right) \\
q_{2}=k_{2}+d \sigma_{2}, & \hat{\mu}_{2}=k_{2} \mu_{2} / q_{2}, & \gamma=\sqrt{q_{2}^{2}+2 \sigma_{2}^{2}}
\end{array}
$$

Numbers in parentheses represent the average of the $t$-statistics of cross-sectional regressions. The numbers in square brackets [.] represent the standard deviation of the time series of parameter estimates.

\begin{tabular}{ccccccc}
\hline & $\mathbf{1 9 9 1 - 1 9 9 5}$ & $\mathbf{1 9 9 1}$ & $\mathbf{1 9 9 2}$ & $\mathbf{1 9 9 3}$ & $\mathbf{1 9 9 4}$ & $\mathbf{1 9 9 5}$ \\
\hline \multirow{4}{*}{$q_{1}$} & 0.6302 & 0.9068 & 0.4618 & 0.8731 & 0.5440 & 0.3577 \\
& $(120.66)$ & $(99.92)$ & $(116.21)$ & $(70.97)$ & $(154.12)$ & $(162.88)$ \\
& {$[0.5376]$} & {$[0.7612]$} & {$[0.2526]$} & {$[0.7065]$} & {$[0.1749]$} & {$[0.1515]$} \\
\hline \multirow{2}{*}{$q_{2}$} & 0.6461 & 0.8175 & 0.3490 & 1.2430 & 0.5823 & 0.2227 \\
& $(87.88)$ & $(92.79)$ & $(77.04)$ & $(117.71)$ & $(104.31)$ & $(46.68)$ \\
& {$[0.6430]$} & {$[0.7786]$} & {$[0.1967]$} & {$[0.7156]$} & {$[0.4736]$} & {$[0.1431]$} \\
\hline \multirow{3}{*}{$s^{*}$} & 0.0939 & 0.1059 & 0.0919 & 0.1010 & 0.0828 & 0.0876 \\
& $(784.10)$ & $(1559.51)$ & $(572.70)$ & $(945.38)$ & $(580.79)$ & $(254.74)$ \\
& {$[0.0170]$} & {$[0.0103]$} & {$[0.0150]$} & {$[0.0081]$} & {$[0.0174]$} & {$[0.0199]$} \\
\hline
\end{tabular}




\section{Table III. Averages of Market Prices of Risk}

This table contains the estimation results, for each day of the period 1991-1995, of the market prices of risk $\left(\lambda_{i}, i=1,2\right)$ related to each state variable in both two-factor models. Numbers in parentheses represent the average of the $t$-statistics of these estimates. The numbers in square brackets [.] represent the standard deviation of the time series of market prices of risk estimates.

Panel A: Vasicek-CIR Model

\begin{tabular}{ccccccc}
\hline & $\mathbf{1 9 9 1 - 1 9 9 5}$ & $\mathbf{1 9 9 1}$ & $\mathbf{1 9 9 2}$ & $\mathbf{1 9 9 3}$ & $\mathbf{1 9 9 4}$ & $\mathbf{1 9 9 5}$ \\
\hline$\lambda_{1}$ & -16.6370 & -25.4189 & -11.1207 & -19.0044 & -15.8773 & -11.6185 \\
$($ Spread $)$ & $(-89.24)$ & $(-89.14)$ & $(-76.07)$ & $(-86.83)$ & $(-118.56)$ & $(-75.26)$ \\
& {$[13.0414]$} & {$[21.2431]$} & {$[7.3795]$} & {$[11.5403]$} & {$[6.1945]$} & {$[5.9674]$} \\
\hline$\lambda_{2}$ & 2.8754 & 3.8128 & 1.5872 & 5.5101 & 2.4110 & 0.9865 \\
(Long rate) & $(85.99)$ & $(91.24)$ & $(74.87)$ & $(116.41)$ & $(102.51)$ & $(44.00)$ \\
& {$[2.9495]$} & {$[3.6390]$} & {$[0.9426]$} & {$[3.4294]$} & {$[1.9340]$} & {$[0.6534]$} \\
\hline
\end{tabular}

Panel B: Vasicek-Vasicek Model

\begin{tabular}{ccccccc}
\hline & $\mathbf{1 9 9 1 - 1 9 9 5}$ & $\mathbf{1 9 9 1}$ & $\mathbf{1 9 9 2}$ & $\mathbf{1 9 9 3}$ & $\mathbf{1 9 9 4}$ & $\mathbf{1 9 9 5}$ \\
\hline$\lambda_{1}$ & 0.2386 & 3.1513 & 1.8632 & 2.6534 & -0.7678 & -5.7401 \\
$($ Spread $)$ & $(5.09)$ & $(46.88)$ & $(34.10)$ & $(1.19)$ & $(-2.07)$ & $(-54.31)$ \\
& {$[12.2793]$} & {$[10.1866]$} & {$[5.6779]$} & {$[13.3915]$} & {$[18.0933]$} & {$[7.5040]$} \\
\hline$\lambda_{2}$ & 4.8419 & -8.4789 & -6.9682 & 37.8514 & -1.7900 & 2.8856 \\
$($ Long rate) & $(15.22)$ & $(-49.31)$ & $(-30.41)$ & $(30.75)$ & $(-29.76)$ & $(1.75)$ \\
& {$[41.8299]$} & {$[32.8720]$} & {$[15.2471]$} & {$[59.6728]$} & {$[44.0095]$} & {$[20.7386]$} \\
\hline
\end{tabular}


Table IV. Within-Sample Pricing Error Measures. 1991-1994

This table contains the within-sample pricing error measures of both two-factor models for the period 1991-1994. We consider zero-coupon bonds with face value of $\$ 1$ and with maturities ranging from 1 day to 10 years. We have computed five different error measures: the mean error (ME), the mean absolute error (MAE), the root mean squared error (RMSE), the mean absolute percentage error (MAPE) and the root mean squared percentage error (RMSPE).

Vasicek-CIR Model

\begin{tabular}{llllll}
\hline Maturity & ME & MAE & RMSE & MAPE & RMSPE \\
\hline 7-day & -0.000000 & 0.000001 & 0.000004 & 0.000083 & 0.000366 \\
15-day & -0.000001 & 0.000002 & 0.000012 & 0.000175 & 0.001214 \\
1-month & -0.000002 & 0.000004 & 0.000034 & 0.000382 & 0.003471 \\
3-month & -0.000001 & 0.000008 & 0.000035 & 0.000803 & 0.003651 \\
6-month & 0.000006 & 0.000013 & 0.000060 & 0.001373 & 0.006402 \\
1-year & 0.000020 & 0.000032 & 0.000115 & 0.003515 & 0.012915 \\
3-year & 0.000003 & 0.000061 & 0.000133 & 0.008256 & 0.018230 \\
5-year & -0.000077 & 0.000119 & 0.000187 & 0.019644 & 0.030982 \\
\hline
\end{tabular}

Vasicek-Vasicek Model

\begin{tabular}{llllll}
\hline Maturity & ME & MAE & RMSE & MAPE & RMSPE \\
\hline 7-day & -0.000000 & 0.000001 & 0.000004 & 0.000106 & 0.000421 \\
15-day & 0.000000 & 0.000003 & 0.000015 & 0.000321 & 0.001519 \\
1-month & 0.000003 & 0.000012 & 0.000053 & 0.001239 & 0.005413 \\
3-month & 0.000034 & 0.000074 & 0.000273 & 0.007631 & 0.028254 \\
6-month & 0.000109 & 0.000200 & 0.000712 & 0.021114 & 0.075686 \\
1-year & 0.000241 & 0.000480 & 0.001457 & 0.053265 & 0.162536 \\
3-year & -0.000081 & 0.000517 & 0.001156 & 0.070541 & 0.159114 \\
5-year & -0.000481 & 0.001547 & 0.002887 & 0.256526 & 0.467754 \\
\hline
\end{tabular}




\section{Table V. Within-Sample Pricing Error Measures. 1992}

This table contains the within-sample pricing error measures of both two-factor models for the year 1992. We consider zero-coupon bonds with face value of $\$ 1$ and with maturities ranging from 1 day to 10 years. We have computed five different error measures: the mean error (ME), the mean absolute error (MAE), the root mean squared error (RMSE), the mean absolute percentage error (MAPE) and the root mean squared percentage error (RMSPE).

Vasicek-CIR Model

\begin{tabular}{llllll}
\hline Maturity & ME & MAE & RMSE & MAPE & RMSPE \\
\hline 7-day & 0.000000 & 0.000000 & 0.000000 & 0.000021 & 0.000033 \\
15-day & 0.000000 & 0.000000 & 0.000001 & 0.000038 & 0.000064 \\
1-month & 0.000001 & 0.000001 & 0.000001 & 0.000084 & 0.000140 \\
3-month & 0.000004 & 0.000004 & 0.000005 & 0.000428 & 0.000551 \\
6-month & 0.000011 & 0.000012 & 0.000015 & 0.001304 & 0.001630 \\
1-year & 0.000023 & 0.000033 & 0.000041 & 0.003671 & 0.004636 \\
3-year & -0.000012 & 0.000034 & 0.000048 & 0.004963 & 0.007023 \\
5-year & -0.000069 & 0.000119 & 0.000146 & 0.020964 & 0.025712 \\
\hline
\end{tabular}

Vasicek-Vasicek Model

\begin{tabular}{llllll}
\hline Maturity & ME & MAE & RMSE & MAPE & RMSPE \\
\hline 7-day & 0.000000 & 0.000000 & 0.000001 & 0.000035 & 0.000068 \\
15-day & 0.000000 & 0.000001 & 0.000002 & 0.000124 & 0.000230 \\
1-month & 0.000001 & 0.000005 & 0.000009 & 0.000533 & 0.000940 \\
3-month & 0.000005 & 0.000037 & 0.000062 & 0.003838 & 0.006357 \\
6-month & 0.000002 & 0.000109 & 0.000188 & 0.011595 & 0.019969 \\
1-year & -0.000042 & 0.000305 & 0.000510 & 0.034493 & 0.057827 \\
3-year & -0.000081 & 0.000349 & 0.000500 & 0.050313 & 0.072872 \\
5-year & 0.000208 & 0.001013 & 0.001542 & 0.181938 & 0.281741 \\
\hline
\end{tabular}


Table VI. Within-Sample Pricing Error Measures for 1-year Bonds

This table contains the within-sample pricing error measures of both two-factor models for each semester of the period 1991-1994. We consider zero-coupon bonds with face value of $\$ 1$ and with maturity of 1 year. We have computed five different error measures: the mean error (ME), the mean absolute error (MAE), the root mean squared error (RMSE), the mean absolute percentage error (MAPE) and the root mean squared percentage error (RMSPE).

Vasicek-CIR Model

\begin{tabular}{llllll}
\hline Period & ME & MAE & RMSE & MAPE & RMSPE \\
\hline 1991:I & 0.000003 & 0.000023 & 0.000030 & 0.002556 & 0.003426 \\
1991:II & 0.000007 & 0.000023 & 0.000033 & 0.002587 & 0.003700 \\
1992:I & 0.000031 & 0.000037 & 0.000045 & 0.004192 & 0.005008 \\
1992:II & 0.000015 & 0.000028 & 0.000037 & 0.003132 & 0.004217 \\
1993:I & 0.000040 & 0.000052 & 0.000308 & 0.005884 & 0.034621 \\
1993:II & 0.000039 & 0.000039 & 0.000045 & 0.004286 & 0.004886 \\
1994:I & 0.000019 & 0.000024 & 0.000029 & 0.002616 & 0.003080 \\
1994:II & 0.000008 & 0.000026 & 0.000047 & 0.002821 & 0.005079 \\
\hline
\end{tabular}

Vasicek-Vasicek Model

\begin{tabular}{llllll}
\hline Period & ME & MAE & RMSE & MAPE & RMSPE \\
\hline 1991:I & -0.000138 & 0.000365 & 0.000708 & 0.041316 & 0.079741 \\
1991:II & 0.000386 & 0.000663 & 0.001082 & 0.074359 & 0.121425 \\
1992:I & -0.000062 & 0.000269 & 0.000428 & 0.030164 & 0.048048 \\
1992:II & -0.000022 & 0.000342 & 0.000584 & 0.038968 & 0.066439 \\
1993:I & 0.000586 & 0.000774 & 0.003241 & 0.086797 & 0.364635 \\
1993:II & 0.000480 & 0.000493 & 0.001365 & 0.053847 & 0.149408 \\
1994:I & 0.000753 & 0.000794 & 0.001435 & 0.085701 & 0.154830 \\
1994:II & -0.000096 & 0.000113 & 0.000444 & 0.012277 & 0.048524 \\
\hline
\end{tabular}


Table VII. Comparison of One-Step-Ahead Forecasts. 1995

This table contains the out-of-sample pricing error measures of both two-factor models for the year 1995. We compute one-step-ahead forecasts for prices of zero-coupon bonds with face value of $\$ 1$ and with maturities ranging from 1 day to 10 years. We report five different error measures: the mean error (ME), the mean absolute error (MAE), the root mean squared error (RMSE), the mean absolute percentage error (MAPE) and the root mean squared percentage error (RMSPE).

Vasicek-CIR Model

\begin{tabular}{llllll}
\hline Maturity & ME & MAE & RMSE & MAPE & RMSPE \\
\hline 7-day & 0.000000 & 0.000016 & 0.000024 & 0.001649 & 0.002412 \\
15-day & 0.000001 & 0.000032 & 0.000047 & 0.003247 & 0.004745 \\
1-month & 0.000002 & 0.000067 & 0.000098 & 0.006782 & 0.009899 \\
3-month & 0.000006 & 0.000176 & 0.000254 & 0.017962 & 0.025969 \\
6-month & 0.000015 & 0.000292 & 0.000417 & 0.030523 & 0.043680 \\
1-year & 0.000036 & 0.000457 & 0.000650 & 0.050227 & 0.071588 \\
3-year & 0.000168 & 0.001273 & 0.001703 & 0.173836 & 0.233587 \\
5-year & 0.000310 & 0.001847 & 0.002397 & 0.315120 & 0.411416 \\
\hline
\end{tabular}

Vasicek-Vasicek Model

\begin{tabular}{llllll}
\hline Maturity & ME & MAE & RMSE & MAPE & RMSPE \\
\hline 7-day & 0.000000 & 0.000016 & 0.000024 & 0.001648 & 0.002410 \\
15-day & 0.000000 & 0.000032 & 0.000047 & 0.003242 & 0.004738 \\
1-month & -0.000001 & 0.000067 & 0.000098 & 0.006781 & 0.009874 \\
3-month & -0.000016 & 0.000180 & 0.000255 & 0.018363 & 0.026064 \\
6-month & -0.000065 & 0.000321 & 0.000436 & 0.033589 & 0.045608 \\
1-year & -0.000209 & 0.000580 & 0.000767 & 0.063822 & 0.084462 \\
3-year & -0.000239 & 0.001320 & 0.001790 & 0.180321 & 0.245460 \\
5-year & 0.000961 & 0.002096 & 0.002760 & 0.357493 & 0.473140 \\
\hline
\end{tabular}


Table VIII. Comparison of One-Step-Ahead Forecasts. 1-year Bonds

This table contains the out-of-sample pricing error measures of both two-factor models for each month of the year 1995. We compute one-step-ahead forecasts for prices of zerocoupon bonds with face value of $\$ 1$ and with maturity of 1 year. We report five different error measures: the mean error (ME), the mean absolute error (MAE), the root mean squared error (RMSE), the mean absolute percentage error (MAPE) and the root mean squared percentage error (RMSPE).

Vasicek-CIR Model

\begin{tabular}{llllll}
\hline Period & ME & MAE & RMSE & MAPE & RMSPE \\
\hline 1995:I & -0.000102 & 0.000830 & 0.001048 & 0.091516 & 0.115583 \\
1995:II & 0.000037 & 0.000592 & 0.000726 & 0.065100 & 0.079775 \\
1995:III & -0.000149 & 0.000758 & 0.000971 & 0.083832 & 0.107340 \\
1995:IV & 0.000044 & 0.000679 & 0.000821 & 0.074892 & 0.090439 \\
1995:V & -0.000086 & 0.000650 & 0.000798 & 0.071588 & 0.087900 \\
1995:VI & 0.000056 & 0.000299 & 0.000367 & 0.032940 & 0.040464 \\
1995:VII & 0.000081 & 0.000171 & 0.000284 & 0.018823 & 0.031279 \\
1995:VIII & 0.000180 & 0.000285 & 0.000396 & 0.031256 & 0.043447 \\
1995:IX & -0.000013 & 0.000339 & 0.000485 & 0.037148 & 0.053150 \\
1995:X & 0.000047 & 0.000187 & 0.000264 & 0.020504 & 0.028900 \\
1995:XI & 0.000147 & 0.000274 & 0.000377 & 0.029984 & 0.041194 \\
1995:XII & 0.000217 & 0.000462 & 0.000650 & 0.050265 & 0.070714 \\
\hline
\end{tabular}

Vasicek-Vasicek Model

\begin{tabular}{llllll}
\hline Period & ME & MAE & RMSE & MAPE & RMSPE \\
\hline 1995:I & -0.000128 & 0.000806 & 0.001028 & 0.088825 & 0.113421 \\
1995:II & 0.000042 & 0.000598 & 0.000736 & 0.065687 & 0.080922 \\
1995:III & -0.000157 & 0.000751 & 0.000966 & 0.082970 & 0.106741 \\
1995:IV & 0.000007 & 0.000642 & 0.000755 & 0.070767 & 0.083297 \\
1995:V & -0.000564 & 0.000861 & 0.000993 & 0.094891 & 0.109457 \\
1995:VI & -0.000553 & 0.000726 & 0.000900 & 0.080044 & 0.099249 \\
1995:VII & -0.000336 & 0.000522 & 0.000727 & 0.057492 & 0.080025 \\
1995:VIII & -0.000311 & 0.000480 & 0.000628 & 0.052822 & 0.069009 \\
1995:IX & -0.000476 & 0.000550 & 0.000713 & 0.060301 & 0.078096 \\
1995:X & -0.000183 & 0.000272 & 0.000346 & 0.029797 & 0.037896 \\
1995:XI & 0.000055 & 0.000278 & 0.000374 & 0.030352 & 0.040852 \\
1995:XII & 0.000233 & 0.000458 & 0.000651 & 0.049808 & 0.070898 \\
\hline
\end{tabular}


Table IX. Comparison of Five-Step-Ahead Forecasts. 1995

This table contains the out-of-sample pricing error measures of both two-factor models for the year 1995. We compute five-step-ahead forecasts for prices of zero-coupon bonds with face value of $\$ 1$ and with maturities ranging from 1 day to 10 years. We report five different error measures: the mean error (ME), the mean absolute error (MAE), the root mean squared error (RMSE), the mean absolute percentage error (MAPE) and the root mean squared percentage error (RMSPE).

Vasicek-CIR Model

\begin{tabular}{llllll}
\hline Maturity & ME & MAE & RMSE & MAPE & RMSPE \\
\hline 7-day & 0.000000 & 0.000029 & 0.000041 & 0.002910 & 0.004115 \\
15-day & 0.000001 & 0.000057 & 0.000081 & 0.005754 & 0.008149 \\
1-month & 0.000003 & 0.000121 & 0.000171 & 0.012148 & 0.017260 \\
3-month & 0.000019 & 0.000327 & 0.000471 & 0.033447 & 0.048175 \\
6-month & 0.000062 & 0.000581 & 0.000855 & 0.060846 & 0.089560 \\
1-year & 0.000194 & 0.001072 & 0.001558 & 0.117955 & 0.171596 \\
3-year & 0.000868 & 0.003318 & 0.004242 & 0.452690 & 0.581056 \\
5-year & 0.001312 & 0.004669 & 0.005904 & 0.795712 & 1.011933 \\
\hline
\end{tabular}

Vasicek-Vasicek Model

\begin{tabular}{llllll}
\hline Maturity & ME & MAE & RMSE & MAPE & RMSPE \\
\hline 7-day & 0.000000 & 0.000029 & 0.000041 & 0.002909 & 0.004112 \\
15-day & 0.000001 & 0.000057 & 0.000081 & 0.005751 & 0.008135 \\
1-month & 0.000001 & 0.000120 & 0.000171 & 0.012137 & 0.017204 \\
3-month & -0.000003 & 0.000327 & 0.000469 & 0.033434 & 0.047936 \\
6-month & -0.000017 & 0.000590 & 0.000857 & 0.061792 & 0.089773 \\
1-year & -0.000052 & 0.001140 & 0.001600 & 0.125428 & 0.176266 \\
3-year & 0.000457 & 0.003296 & 0.004247 & 0.449916 & 0.582409 \\
5-year & 0.001967 & 0.004852 & 0.006077 & 0.826338 & 1.039909 \\
\hline
\end{tabular}


Table X. Comparison of Five-Step-Ahead Forecasts. 1- and 5-year Bonds

This table contains the out-of-sample pricing error measures of both two-factor models for each quarter of the year 1995. We compute five-step-ahead forecasts for prices of zerocoupon bonds with face value of $\$ 1$ and with maturity of 1 and 5 years. We report five different error measures: the mean error (ME), the mean absolute error (MAE), the root mean squared error (RMSE), the mean absolute percentage error (MAPE) and the root mean squared percentage error (RMSPE).

Panel A: 1-year Bonds

\begin{tabular}{llllll}
\hline \multicolumn{7}{c}{ Vasicek-CIR Model } \\
\hline Period & ME & MAE & RMSE & MAPE & RMSPE \\
\hline 1995:I & -0.000505 & 0.001652 & 0.002308 & 0.182288 & 0.255000 \\
1995:II & 0.000180 & 0.001082 & 0.001431 & 0.119313 & 0.157693 \\
1995:III & 0.000447 & 0.000698 & 0.000985 & 0.076596 & 0.108032 \\
1995:IV & 0.000667 & 0.000860 & 0.001161 & 0.093834 & 0.126462 \\
\hline \multicolumn{6}{c}{ Vasicek-Vasicek Model } \\
\hline Period & ME & MAE & RMSE & MAPE & RMSPE \\
\hline 1995:I & -0.000513 & 0.001642 & 0.002300 & 0.181183 & 0.254149 \\
1995:II & -0.000186 & 0.001326 & 0.001612 & 0.146189 & 0.177747 \\
1995:III & -0.000005 & 0.000761 & 0.001005 & 0.083563 & 0.110399 \\
1995:IV & 0.000520 & 0.000836 & 0.001153 & 0.091139 & 0.125664 \\
\hline
\end{tabular}

Panel B: 5-year Bonds

\begin{tabular}{llllll}
\hline \multicolumn{5}{c}{ Vasicek-CIR Model } \\
\hline Period & ME & MAE & RMSE & MAPE & RMSPE \\
\hline $1995:$ I & -0.001216 & 0.004647 & 0.006103 & 0.822578 & 1.086229 \\
$1995:$ II & 0.001559 & 0.005908 & 0.007249 & 1.021432 & 1.251052 \\
$1995:$ III & 0.001996 & 0.004360 & 0.005489 & 0.729830 & 0.918156 \\
$1995:$ IV & 0.002977 & 0.003765 & 0.004417 & 0.609400 & 0.711505 \\
\hline \multicolumn{6}{c}{ Vasicek-Vasicek Model } \\
\hline Period & ME & MAE & RMSE & MAPE & RMSPE \\
\hline $1995:$ I & -0.000945 & 0.004740 & 0.006169 & 0.838764 & 1.097282 \\
$1995:$ II & 0.002615 & 0.005902 & 0.007305 & 1.019201 & 1.258717 \\
$1995:$ III & 0.003061 & 0.004897 & 0.005972 & 0.820306 & 1.000055 \\
$1995:$ IV & 0.003184 & 0.003853 & 0.004517 & 0.623691 & 0.727507 \\
\hline
\end{tabular}




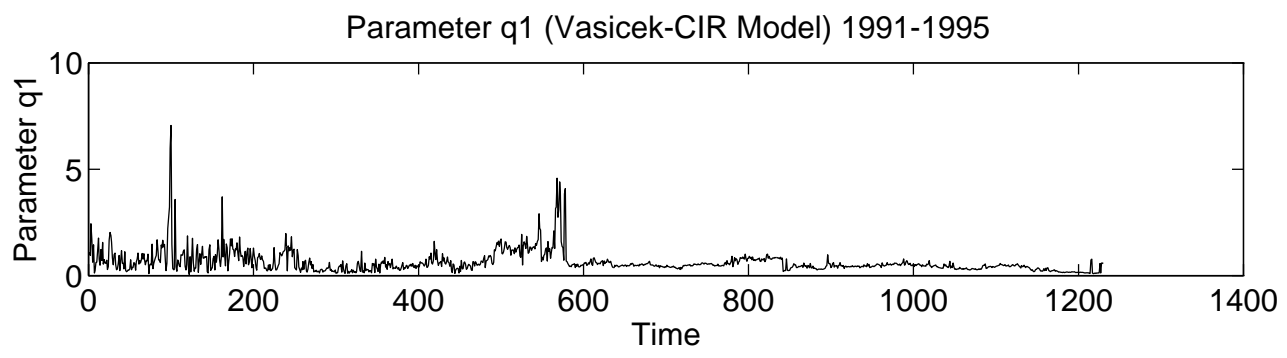

Parameter q2 (Vasicek-CIR Model) 1991-1995

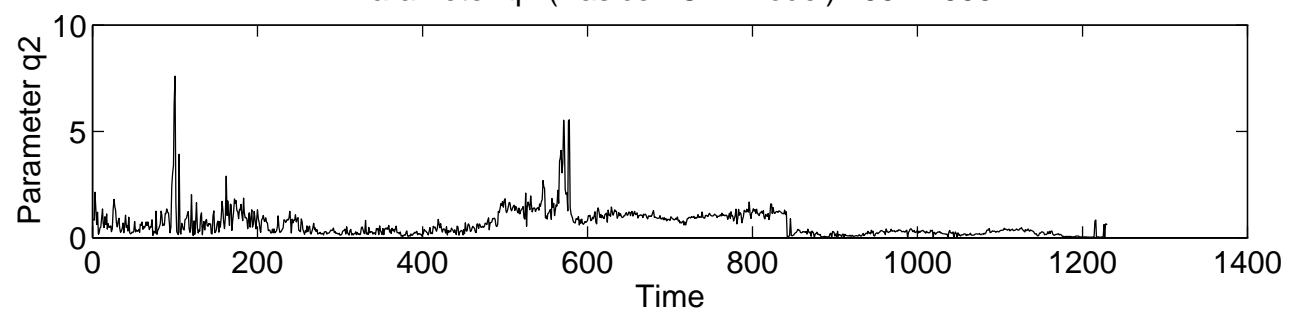

Parameter s* (Vasicek-CIR Model) 1991-1995

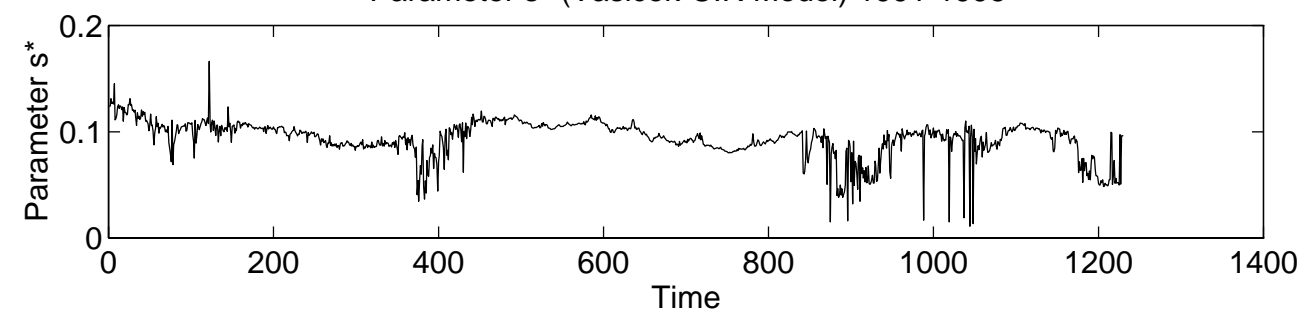

Figure 1: Plot of pure-cross parameters in the Vasicek-CIR model. 

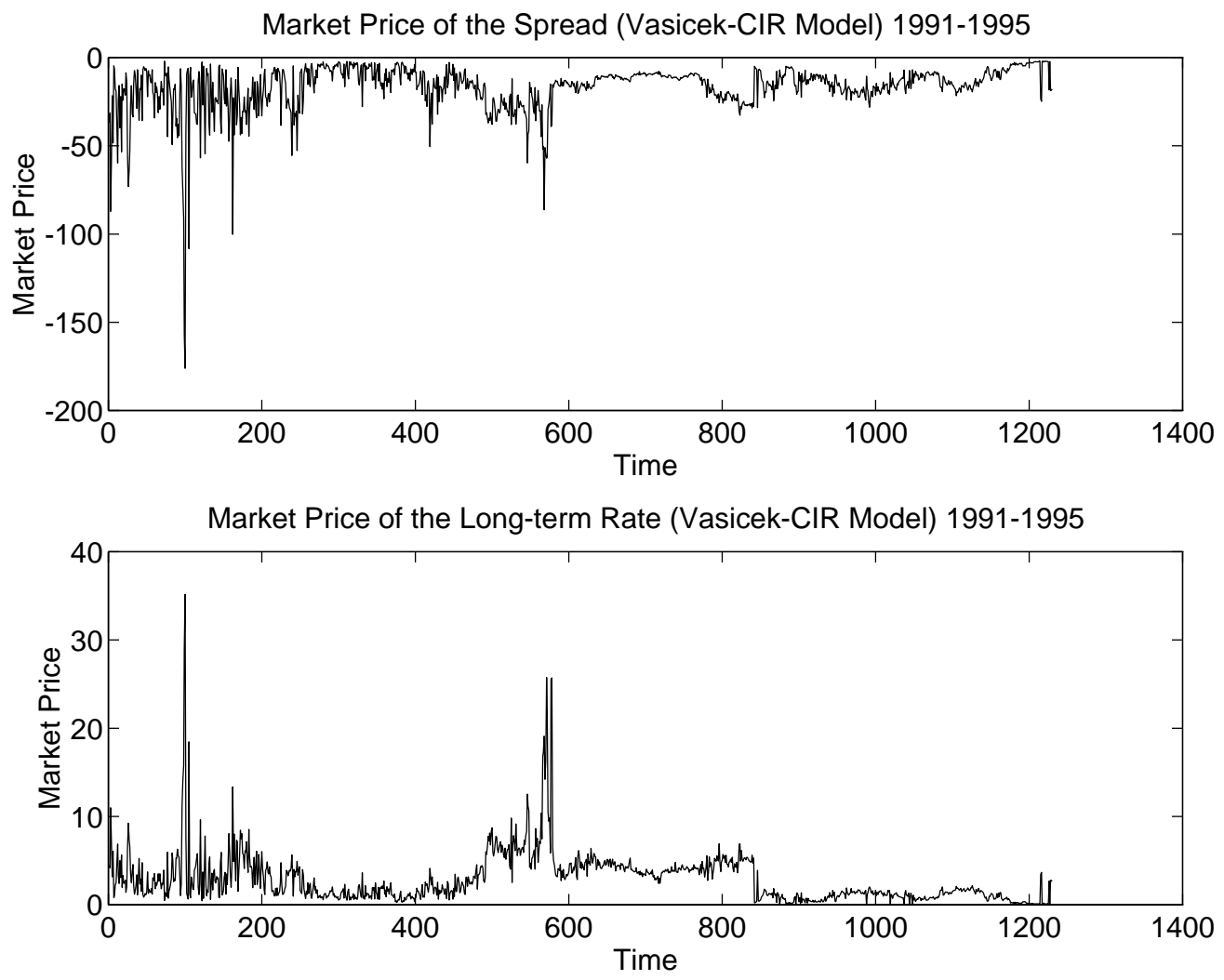

Figure 2: Plot of market prices of risk in the Vasicek-CIR model. 

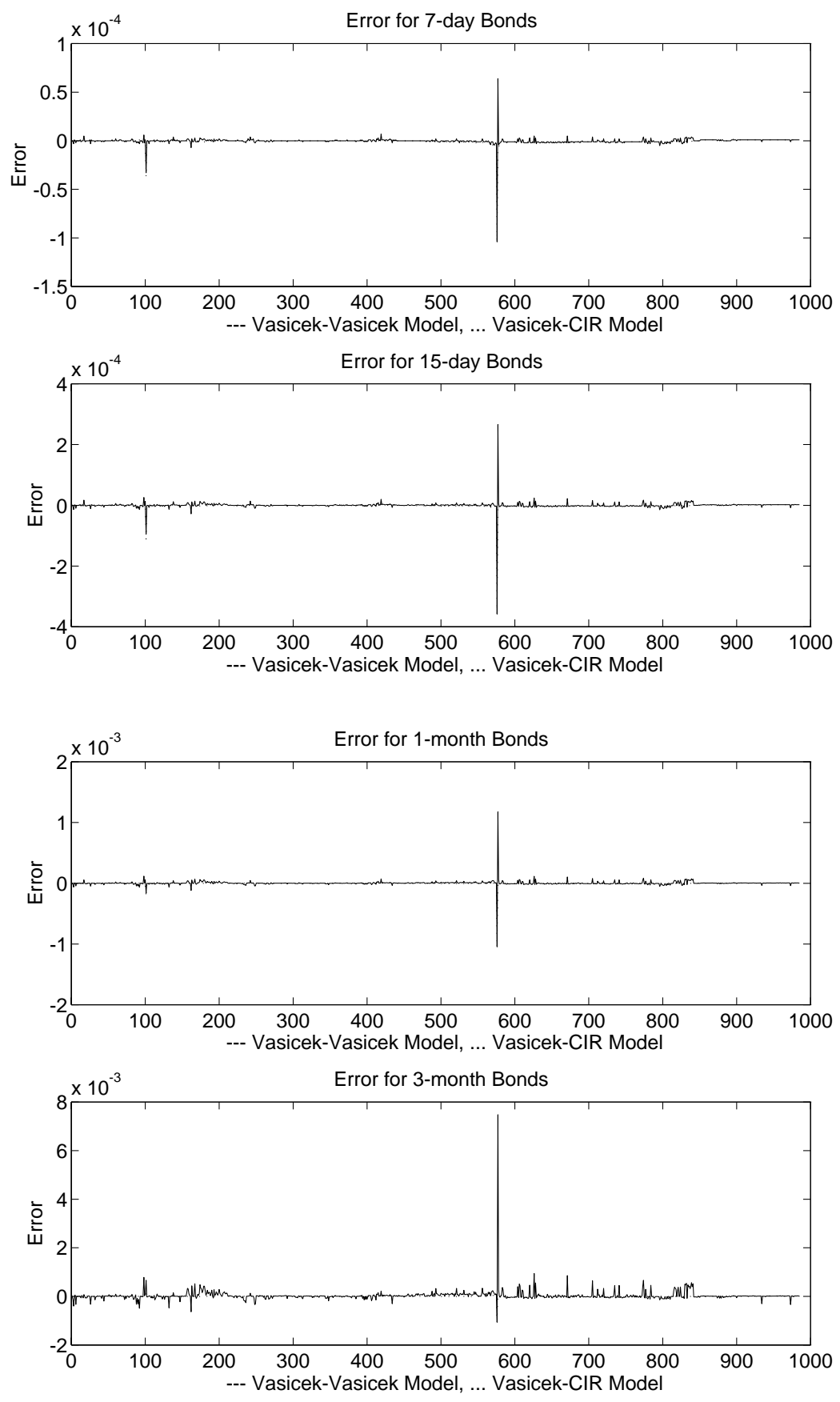

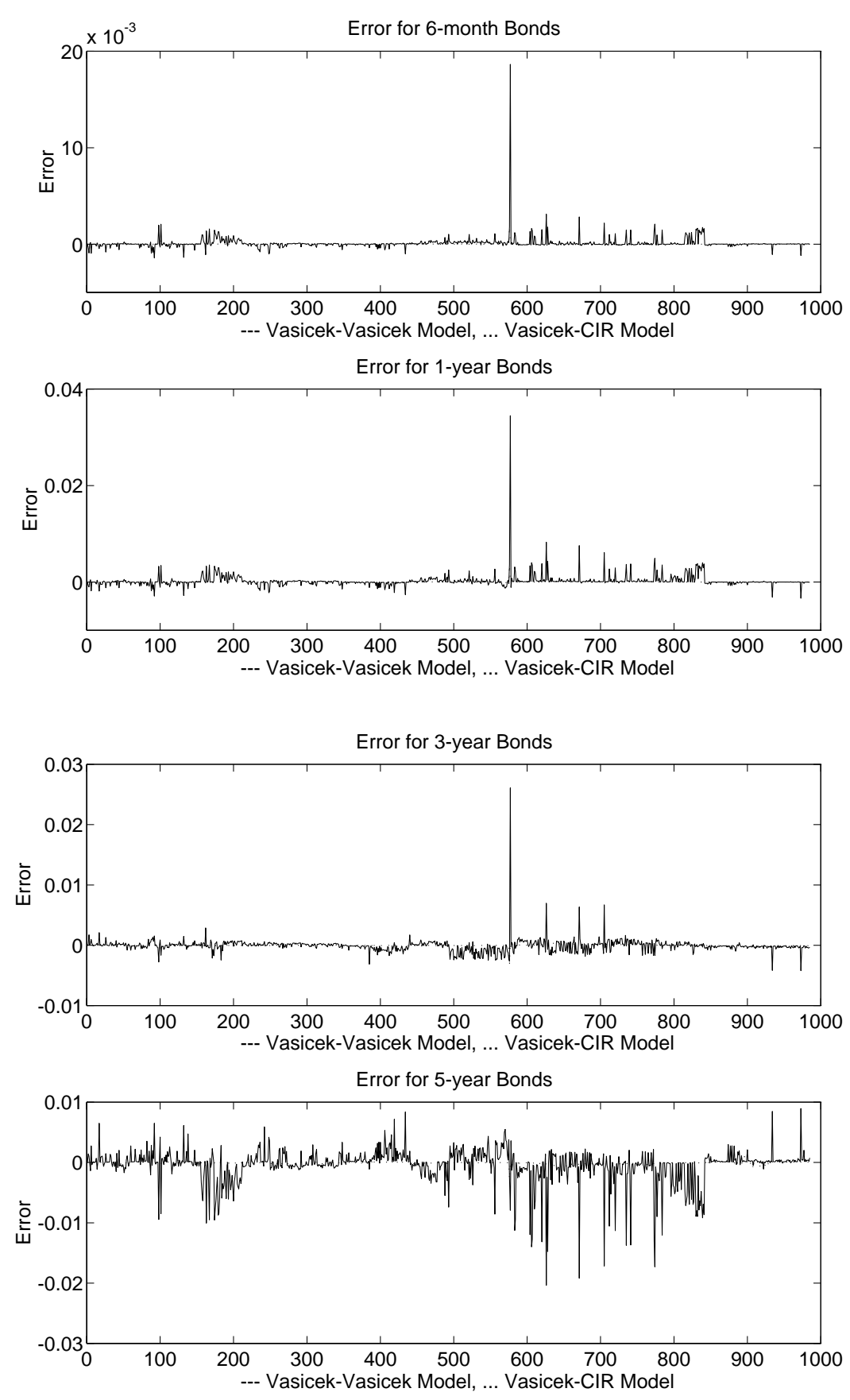

Figure 3: Within-Sample Errors of the Vasicek-Vasicek and Vasicek-CIR Models. 


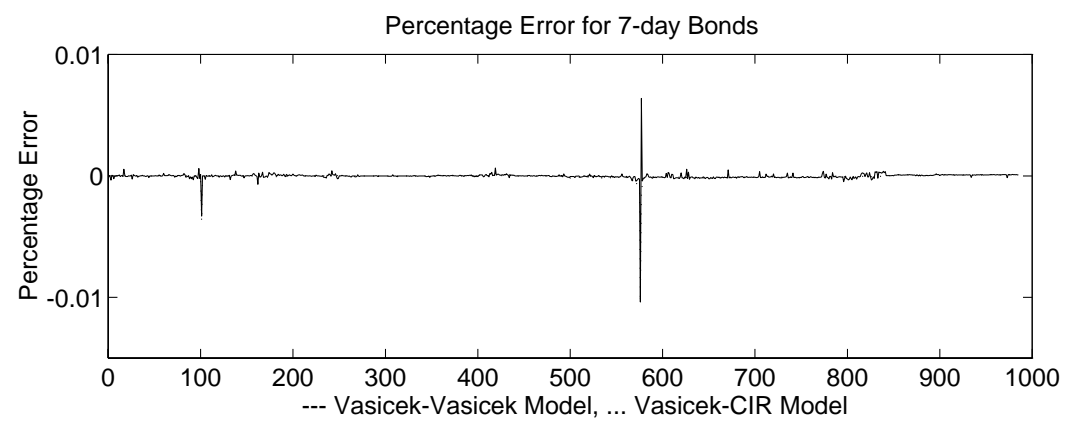

Percentage Error for 15-day Bonds

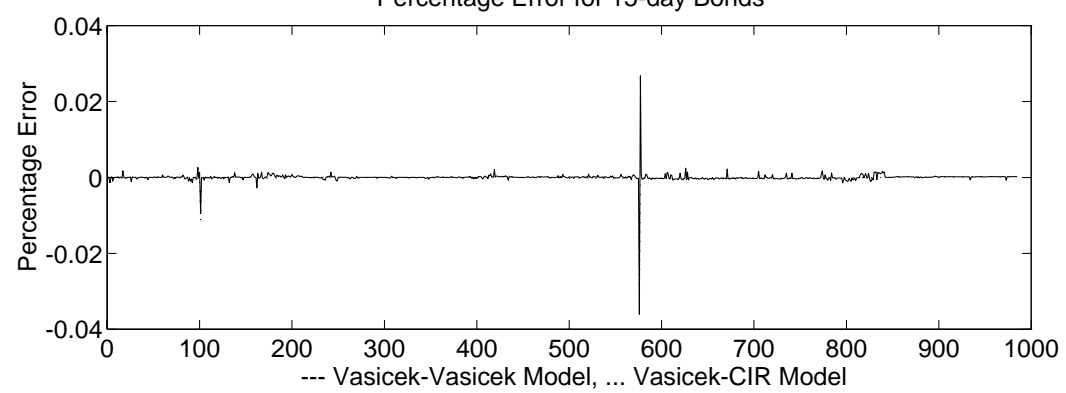

Percentage Error for 1-month Bonds

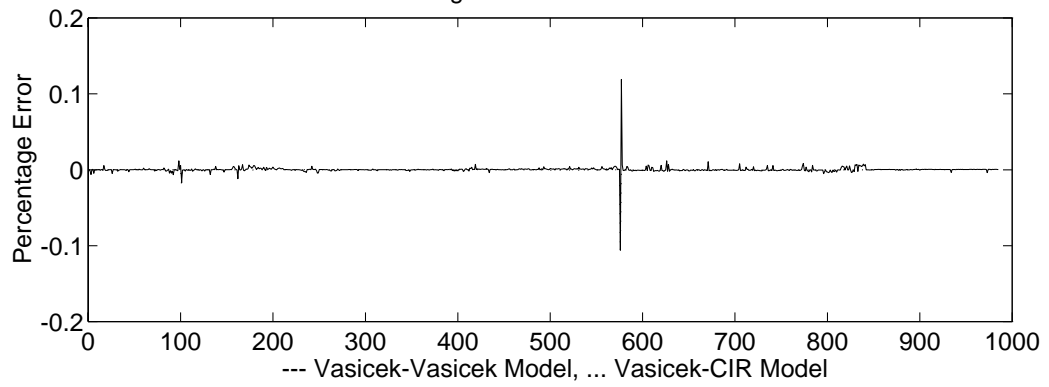

Percentage Error for 3-month Bonds

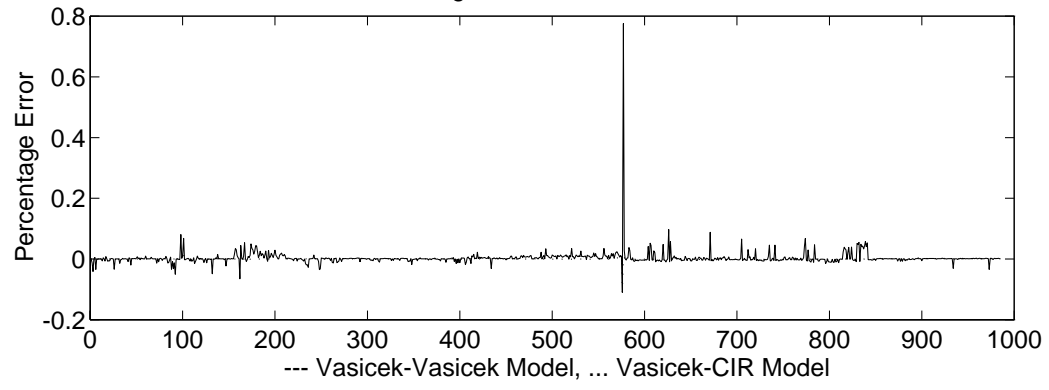



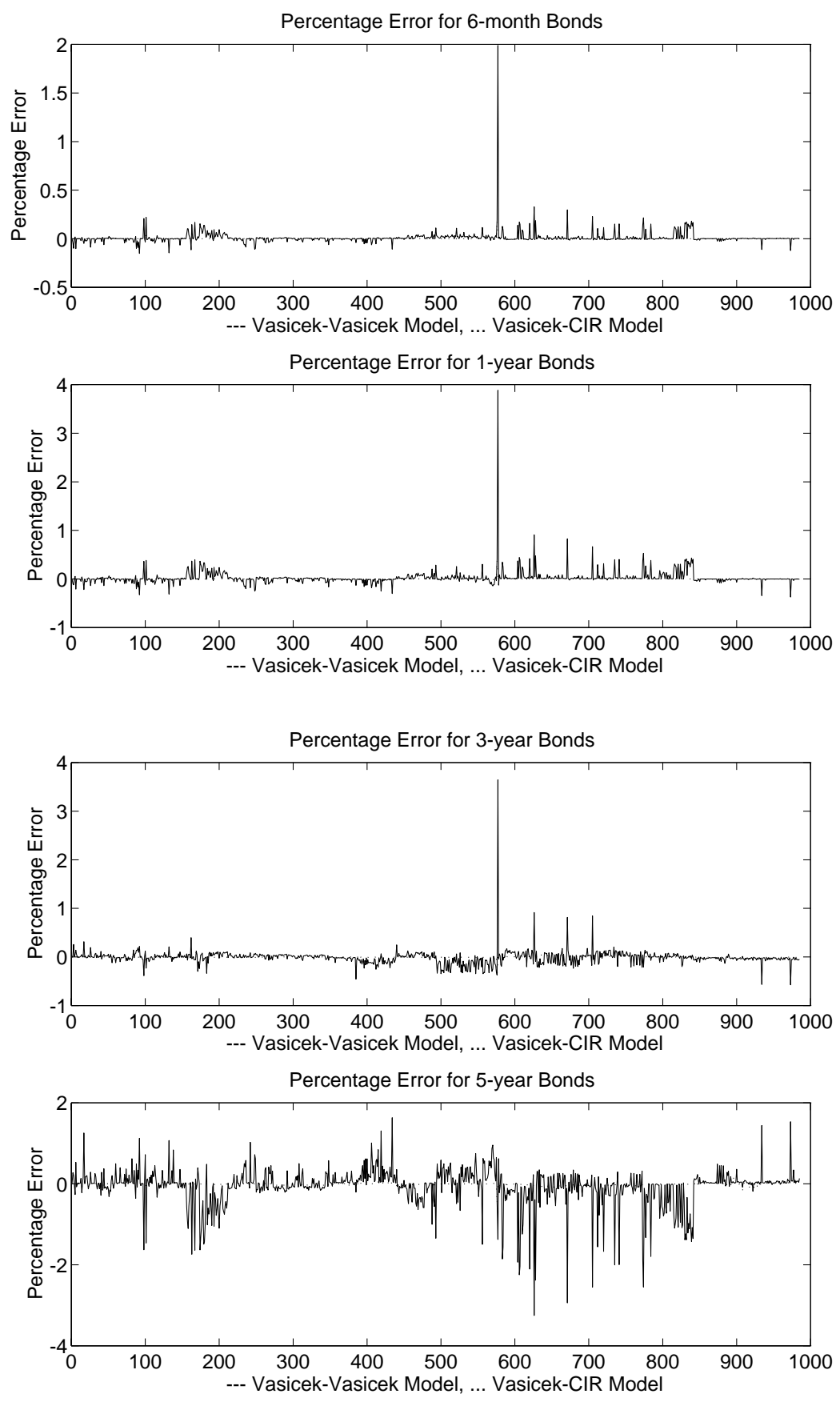

Figure 4: Within-Sample Percentage Errors of the Vasicek-Vasicek and Vasicek-CIR Models. 